$\mathbb{T}$ Periodica Polytechnica Electrical Engineering and Computer Science

60(3), pp. 178-186, 2016

DOI: $10.3311 /$ PPee.9310

Creative Commons Attribution (i)

RESEARCH ARTICLE

\section{Characterization of the Inverse Problem in Critical Dimension Measurement of Diffraction Gratings}

\author{
Károly Marák ${ }^{1 *}$
}

Received 07 April 2016; accepted after revision 08 June 2016

\begin{abstract}
In this paper, the inverse problem of extracting critical dimensions of the grating is defined, using data obtained by ellipsometric spectrometry. We give an overview of theoretical models describing diffraction gratings and their interactions with incident light, with a special emphasis on the coupledwave method. A method for mapping the output space (points on Poincarés sphere defined by the ellipsometric angles for each wavelength) to the input space (grating dimensions) is presented, where samples of the output space are picked equidistantly. Using this method, distribution of the measurement precision for a given type of experimental setup is established, and tested on examples from a set of permalloy gratings.
\end{abstract}

\section{Keywords}

inverse problem, diffraction grating, coupled-wave method

\footnotetext{
${ }^{1}$ Department of Broadband Infocommunication(s) and Electromagnetic Theory, Faculty of Electrical Engineering and Informatics,

Budapest University of Technology and Economics

${ }^{*}$ Corresponding author, e-mail: marak@hvt.bme.hu
}

\section{Introduction}

Diffraction gratings have been used in spectroscopy and other studies of electromagnetic phenomena for nearly two centuries [1, 2]. Josef Fraunhofer (1798-1826), the discoverer of the dark lines in the solar spectrum, built the first gratings in 1819 by winding fine wires around two parallel screws [3]. Simply said, a diffraction grating is an optical component with a periodic structure used to split and diffract light into beams traveling in different directions. Since these directions depend on the frequency of the incident electromagnetic wave, it can be used as a dispersive element. Because of this, gratings are often used in monochromators and spectrometers.

Modern gratings may have up to thousands of lines per millimeter with near-perfect periodicity [4]. The groove shapes can be controlled to be sinusoidal, rectangular and so forth; these gratings can be made on various metal, plastic and glass substrates and coated with thin-film metal or dielectric stacks. The primary applications of diffraction gratings are in spectroscopy $[1,2]$, (where they are used for analyzing the frequency content of electromagnetic radiation), but they are also used as wavelength selectors in tunable lasers [5], beam sampling mirrors in high-power lasers, band-pass filters, pulse compressors and polarization-sensitive optics, among other applications [6].

Diffraction gratings can be studied by a number of experimental methods, like Atomic Force Microscopy (AFM), Scanning Tunneling Microscopy (STM), Spectroscopic Ellipsometry (SE) [7] or the case of magnetic materials, the Magnetooptical Spectroscopic Scatterometry (MOSS) [8,9] measurements.

The former two methods are are able to study a surface directily, but have their limitations: the usage of STM requires samples to have conductive surface or be further coated by an ultrathin metallic layer, which is a destructive operation disabling the sample's later application, while possible altering the fine structure of the grating patterns. While AFM can be used to investigate any solid material, its accuracy is limited by the tipsample scanning [10]. The latter two are non destructive, do not require direct physical contact with the examined surface, but require more complicated numerical processing; the problem is similar to the inverse problem of atomic level investigation of 


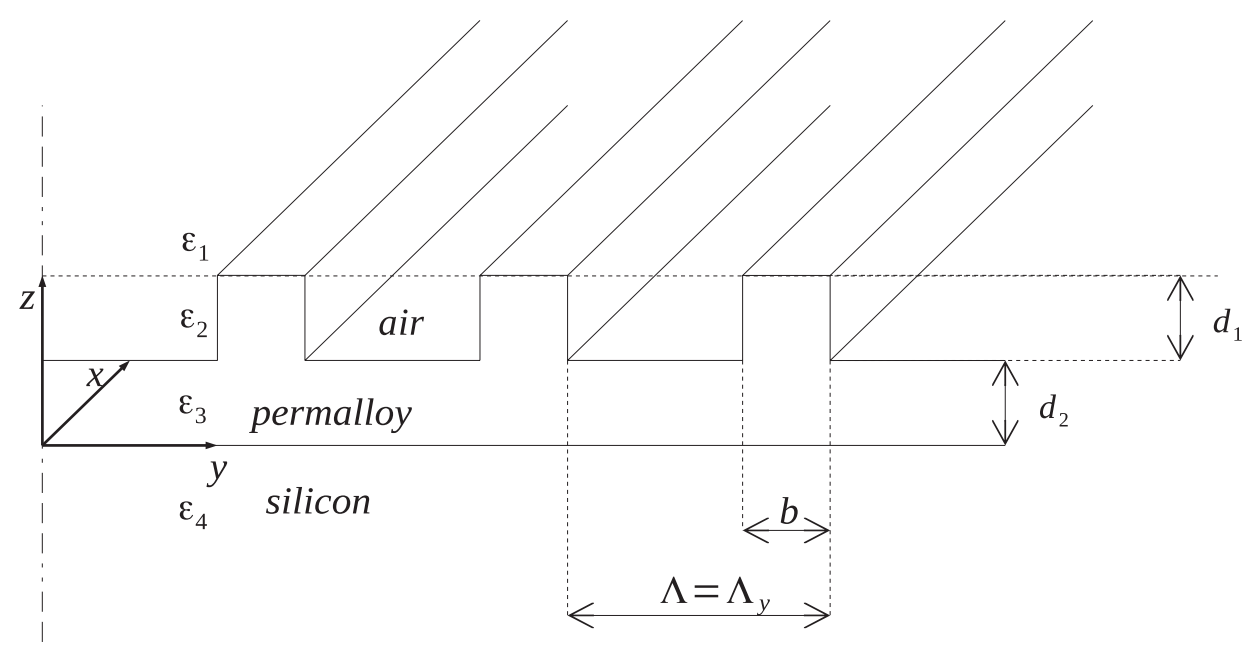

Fig. 1 The geometrical situation of the permalloy grating

solid surfaces and states. Therein, the goal of the investigation is also to get detailed information about the solid surface and state, but specifically at the atomic scale using for example ion scattering, X-ray scattering, low energy electron scattering or thermal energy atomic scattering [11].

Only during the past four decades or so that a thorough understanding of diffraction gratings has been achieved through the consistent application of Maxwell's equations with the help of advanced analytical and numerical techniques [12].

\section{The inverse problem}

\subsection{Definition of the inverse problem}

In general, when we have an operator $f$ mapping one space $(\mathbb{X})$ to another $(\mathbb{Y})$ :

$$
f(\mathbf{x})=\mathbf{y}
$$

we call the task of finding $\mathbf{x}$ for a given $\mathbf{y}$ an inverse problem:

$$
\mathbf{y}=\mathbf{y}_{\mathrm{p}}, \mathbf{x}=\text { ? }
$$

The coupled wave method (see Section 3.1) is able to, for known grating parameters, characterize the reflected light (angle, intensity, polarization of the Fourier-modes). In this work, we use it to calculate the ellipsometric angles of the reflected light from material and geometrical parameters; this is our $f$ forward operator. Specifically, $\mathbf{x}$ is the input space consisting of critical dimensions of the grating and $\mathbf{y}$ is the output space consisting of the measured ellipsometric angles.

It seems plausible to use the formalism to calculate the grating's parameters from observing the reflected light using the aforementioned methods (MOSS, ES); in this case, the inverse problem has to be solved. This has been done for both material and geometric parameters [7, 13].

When working with the inverse problem, we assume that the material parameters (they can be measured easily with a reference flat sample) and grating period $\left(\Lambda=\left(\Lambda_{x}, \Lambda_{y}\right.\right.$, $\Lambda_{z}$ )) are known (since it is relatively easy to control during manufacturing), and that the sum $d_{1}+d_{2}$ is fixed (see Fig. 1 for details) [14]. This leaves $d_{1}$ and $b$ as independent input variables.

$$
\mathbf{x}=\left(d_{1}, b\right), \mathbf{y}=\left(\begin{array}{lll}
\Psi_{1}, & \ldots, & \Psi_{n_{\lambda}} \\
\Delta_{1}, & \ldots, & \Delta_{n_{\lambda}}
\end{array}\right),
$$

where $n_{\lambda}$ is the number of wavelengths at which the experiment is performed, and $\Psi_{i}$ and $\Delta_{i}$ are the ellipsometric angles corresponding to the $\mathrm{i}$-th wavelength examined.

The situation shows some similarities with the problem of Eddy Current Testing (ECT). In that nondestructive testing method (see, e.g., in [15]), the inner structure of conducting materials is examined by applying an incident time-varying magnetic field to generate eddy-currents within the specimen. The reaction field (due to the eddy-currents) is measured by a probe. The measured data are then used to reconstruct the conductivity distribution inside the specimen, which bears information on a possible structural degradation (crack, void, etc.). In our work, the method developed for ECT inverse problems in [16] is used. This algorithm consists in the adaptive generation of a database of corresponding input parameter - output data pairs:

$$
\mathbb{D}=\left\{\left(\mathbf{x}_{1}, \mathbf{y}_{1}\right), \ldots,\left(\mathbf{x}_{s}, \mathbf{y}_{s}\right)\right\},
$$

with $s$ being the total sample number and the pairs are related via the simulation $f(\cdot)$ at hand, i.e.

$$
y_{i}=f\left(\mathbf{x}_{i}\right), \quad i=1,2, \ldots, s .
$$

This database $\mathbb{D}$ is called optimal in the sense that the output samples $\mathbf{y}_{i}$ are uniformly spread out over the output space, i.e., over the domain spanned by all conceivable outputs of the simulation. This uniform output distribution is achieved by means of a sequential adaptive sampling that is not discussed herein. The distribution of the input samples corresponding to such uniform output distribution bears valuable information on the conditioning of the inverse problem, as pointed out in $[16$, 17]. A central contribution of the present paper is to apply this 
adaptive sampling to diffraction gratings and to analyze the available information of the related inverse problem.

The error between a measurement and a calculated value can be characterized by angular distance between the measured and simulated points plotted on Poincaré's sphere [7]. This distance is specified by the azimuthal angle $2 \Psi$ and the polar angle $\Delta$, i.e.,

$$
\cos \gamma=\mathbf{S}_{\text {exp }} \cdot \mathbf{S}_{\text {sim }} .
$$

Here the experimental data is:

$$
\mathbf{S}_{\exp }=\left[\begin{array}{c}
\sin \left(2 \Psi_{\exp }\right) \cos \left(\Delta_{\text {exp }}\right) \\
\sin \left(2 \Psi_{\exp }\right) \sin \left(\Delta_{\exp }\right) \\
\cos \left(2 \Psi_{\exp }\right)
\end{array}\right],
$$

and the simulated data is:

$$
\mathbf{S}_{\text {sim }}=\left[\begin{array}{c}
\sin \left(2 \Psi_{\text {sim }}\right) \cos \left(\Delta_{\text {sim }}\right) \\
\sin \left(2 \Psi_{\text {sim }}\right) \sin \left(\Delta_{\text {sim }}\right) \\
\cos \left(2 \Psi_{\text {sim }}\right)
\end{array}\right] .
$$

Using synthetic data corrupted by a Gaussian noise (this is reasonable to assume, given that the measurement setup is a null-zone ellipsometer [14, 18], which measures the angles themselves [19]), fits were done for several virtual measurements. The aim was to generate output data with noise and try to find the original input by minimizing the sum of errors squared (so the Euclidean norm) over a certain number of measurements on different wavelengths, then to find the relationship between the error of the fit and the density of states on the output map.

$$
\mathbf{x}_{\mathrm{fit}}=\arg \min _{\mathbf{x}}\|\gamma(\tilde{\mathbf{y}}, f(\mathbf{x}))\|^{2},
$$

here $\gamma$ is a vector containing angles $\gamma$ as defined by Eq. (6), and $f$ is the forward operator.

The optimization problem is solved by different numerical techniques: a simplex-method (implemented in the Matlab fmincon-routine, [20]) and a nearest neighbor interpolation over the optimal database are used, as detailed in the numerical examples in Section 3.

\subsection{Characterization of the conditioning of the inverse problem}

Inverse problems can often be ill-posed or ill-conditioned. The latter results in difficulties when solving the optimization problem defined in Eq. (9). In this section, we introduce a certain measure of the ill-conditioning of the inverse problem, namely, the misfit of the found and ,true" solution.

One can calculate the deviation between the input data and the results of the fit:

$$
\left[d_{1, \mathrm{fit}}, b_{\mathrm{fit}}\right]-\left[d_{1}, b\right]=\left[\delta_{d_{1}}, \delta_{b}\right] .
$$

It can be expected that the accuracy of the fitting is better in places where the density of states is larger, and conversely, in places of smaller density, the accuracy is worse. This is proven to be true in later parts of this work.

\section{Grating theories}

To be able to work on the inverse problem, an adequate method is required to arrive to the forward function $f$. There are several theoretical approaches dealing with the EM theory of gratings. The simplest theory of gratings treats them as corrugated structures that modulate the amplitude and/or phase of the incident beam. The modulated reflected (or transmitted) wavefront is then decomposed into its Fourier spectrum to yield the various diffracted orders.

From a mathematical point of view, Maxwell's equations can be expressed as partial differential equations. In the grating case, they have constant coefficients - they can be solved by integrating numerically in two dimensions. Because of numerical instabilities, this method only works for shallow gratings [21].

The integral method is based on the idea that the solution of electromagnetic scattering problems can be found by simple integration, provided that the field and its normal derivative on the surface of the scattering object (and at infinity) are known.

Finite element schemes can also be used. The advantage of them is the availability in commercial software, however, the computational cost involved is usually high.

Modal methods, like the one we're describing in this work, work for lamellar profiles. They are based on the decomposition of EM field in a combination of modes, which are some base vectors chosen according to the situation.

For more details about the methods used to describe diffraction gratings, see [21].

\subsection{The coupled-wave method for a grating with a single anisotropic inhomogeneous region between two isotropic homogeneous regions}

The analytical formalism of Rokushima and Yamakita treating the Fraunhofer diffraction in planar multilayer periodic structures is a powerful method of describing diffraction gratings [22]. Using parameters of the grating (material parameters, geometry) and assuming that the characteristics of the incident light are known, we are able to calculate the intensity, polarization, angles of the refracted and transmitted light in specific diffraction orders. To characterize the grating's geometry, we use that the permittivity tensor inside the grating is periodic (an illustration of the geometric situation can be seen in Fig. 2):

$$
\vec{\varepsilon}(\mathbf{r})=\left(\begin{array}{lll}
\varepsilon_{x x}(\mathbf{r}) & \varepsilon_{x y}(\mathbf{r}) & \varepsilon_{x z}(\mathbf{r}) \\
\varepsilon_{y x}(\mathbf{r}) & \varepsilon_{y y}(\mathbf{r}) & \varepsilon_{y z}(\mathbf{r}) \\
\varepsilon_{z x}(\mathbf{r}) & \varepsilon_{z y}(\mathbf{r}) & \varepsilon_{z z}(\mathbf{r})
\end{array}\right),
$$

while also assuming that the the permeability of the grating is equal to that of vacuum. 


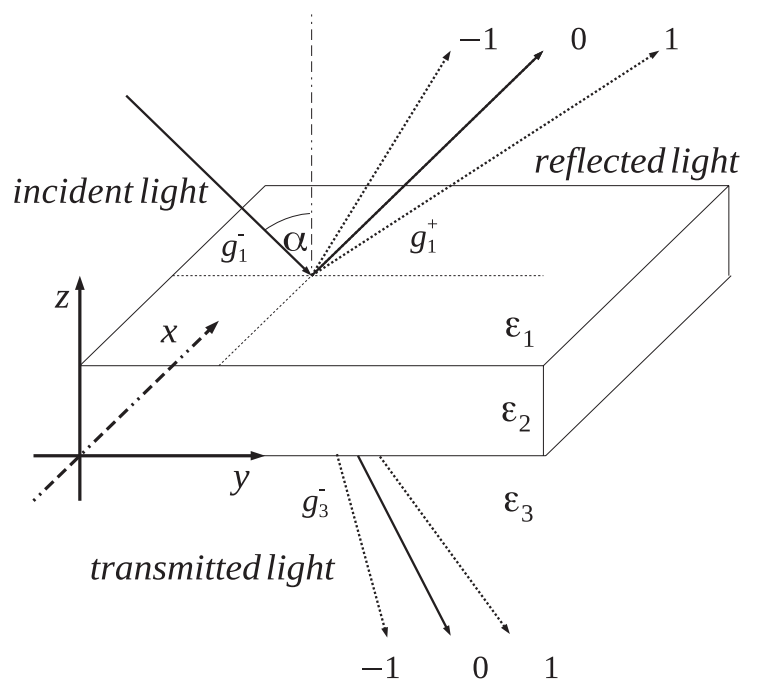

Fig. 2 Geometry and definitions shown on a general diffraction grating. Regions (1) and (3) are homogeneous and isotropic, while (2) can be inhomogeneous anisotropic with a periodic tensor of permittivity.

In the general case, we can characterize the periodicity of the grating region with the so-called grating vector $\boldsymbol{\Gamma}$, which can be understood as a reciprocal lattice to the lattice defined by vector $\Lambda$.

$$
\Gamma=\hat{\mathbf{x}} \Gamma_{x}+\hat{\mathbf{y}} \Gamma_{y}+\hat{\mathbf{z}} \Gamma_{z},
$$

where

$$
\Gamma_{i}=\frac{2 \pi}{\Lambda_{i}}, i=x, y, z
$$

Since $\vec{\varepsilon}(\mathbf{r})$ is periodic, it is possible to expand it into a Fourier series:

$$
\varepsilon_{k l}(\mathbf{r})=\sum_{m} \exp (\jmath l \Gamma \cdot \mathbf{r}) \varepsilon_{k l, m}
$$

or rewriting it with normalized components.

According to the Floquet theorem, the electric and magnetic fields of electromagnetic waves can be expressed as a Fourier series:

$$
\begin{aligned}
& \sqrt{\frac{\mu_{v a c}}{\varepsilon_{v a c}}} \mathbf{E}(\bar{z})=\sum_{m} \mathbf{e}_{m}(\bar{z}) \exp \left(-\jmath \mathbf{n}_{m} \cdot \overline{\mathbf{r}}\right), \\
& \sqrt{\frac{\mu_{v a c}}{\varepsilon_{v a c}}} \mathbf{H}(\bar{z})=\sum_{m} \mathbf{h}_{m}(\bar{z}) \exp \left(-\jmath \mathbf{n}_{m} \cdot \overline{\mathbf{r}}\right),
\end{aligned}
$$

where

$$
\begin{gathered}
\mathbf{e}_{m}(\bar{z})=\hat{\mathbf{x}} e_{x m}(\bar{z})+\hat{\mathbf{y}} e_{y m}(\bar{z})+\hat{\mathbf{z}} e_{z m}(\bar{z}), \\
\mathbf{h}_{m}(\bar{z})=\hat{\mathbf{x}} h_{x m}(\bar{z})+\hat{\mathbf{y}} h_{y m}(\bar{z})+\hat{\mathbf{z}} h_{z m}(\bar{z}), \\
\overline{\mathbf{r}}=\frac{\omega}{c} \mathbf{r}, \quad \bar{z}=\frac{\omega}{c} z,
\end{gathered}
$$

and $\mathbf{n}_{m}$ is a vector dependent on the angle of incidence in addition to the aforementioned grating parameters.

After some mathematical operations and physical considerations detailed in [23], we get the following equations:

$$
\begin{gathered}
\frac{d}{d \bar{z}} \mathbf{f}_{\hat{t}}=\jmath \mathbf{C} \mathbf{f}_{\hat{\imath}}, \\
\mathbf{f}_{\hat{n}}=\mathbf{C} \mathbf{f}_{\hat{\imath}} .
\end{gathered}
$$

Here,

$$
\begin{gathered}
\mathbf{f}_{t}=\left(\begin{array}{l}
e_{x} \\
h_{y} \\
e_{y} \\
h_{x}
\end{array}\right), \\
\mathbf{f}_{n}=\left(\begin{array}{l}
e_{z} \\
h_{z}
\end{array}\right),
\end{gathered}
$$

and $\mathbf{C}$ is a square matrix with a size of $4 \mathrm{Mx} 4 \mathrm{M}$ (where $\mathrm{M}$ is the order of the Fourier series) dependent on the angle of incidence, material and geometric parameters. The solution to Eqs. (20), (21) reduces to a problem of finding the eigensystem of matrix $\mathbf{C}$.

Ultimately, all the information about the grating can be expressed using the transfer matrix (superscripts indicate the region or boundary which the matrices characterize):

$$
\mathbf{W}=\mathbf{B}^{(12)} \mathbf{U}^{(2)}\left(\bar{d}_{2}\right) \mathbf{B}^{(23)} \text {. }
$$

Where $\mathbf{B}^{(12)}$ and $\mathbf{B}^{(23)}$ characterize effects on the respective layers' boundaries, while $\mathbf{U}^{(2)}$ characterizes propagation inside region (2).

\subsection{Rectangular permalloy grating on a semi-infinite layer of silicon}

A certain diffraction grating has been studied extensively using SE and MOSS at the Division of Magnetooptics, Charles University in Prague $[14,18]$. The geometrical situation can be seen in Fig. 1, while the results of the conducted AFM measurement in Fig. 3.

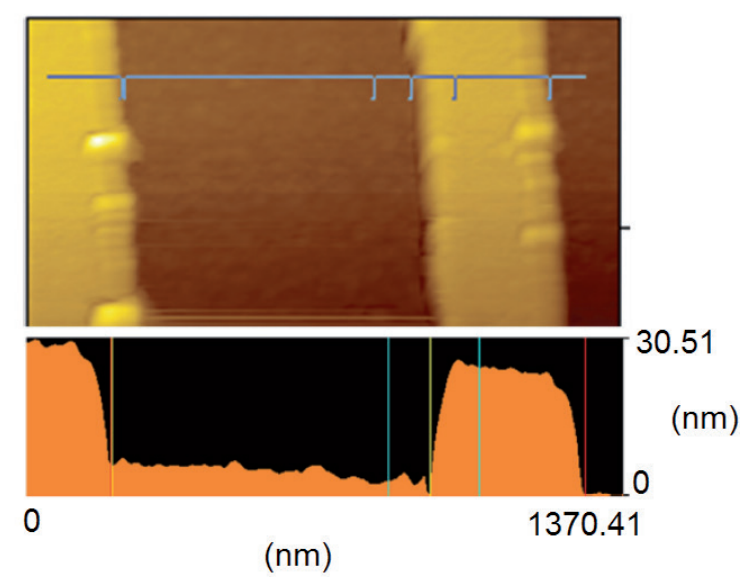

Fig. 3 The AFM measurements 

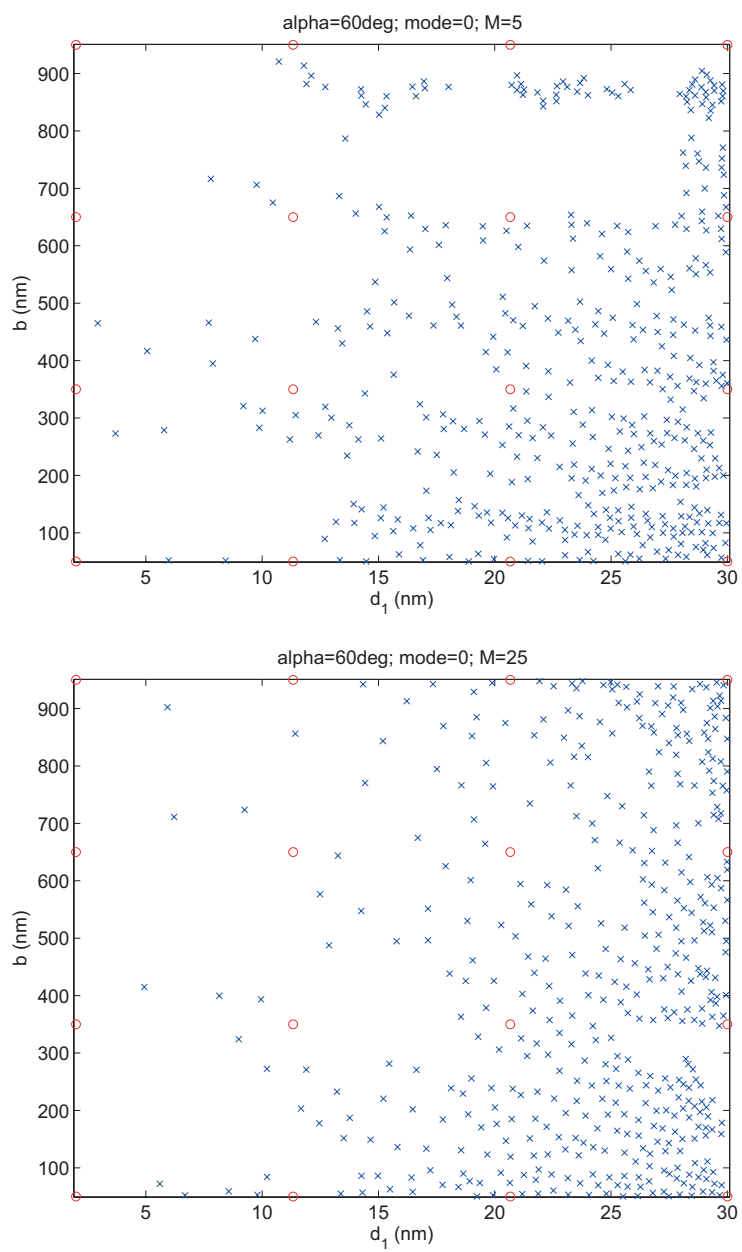
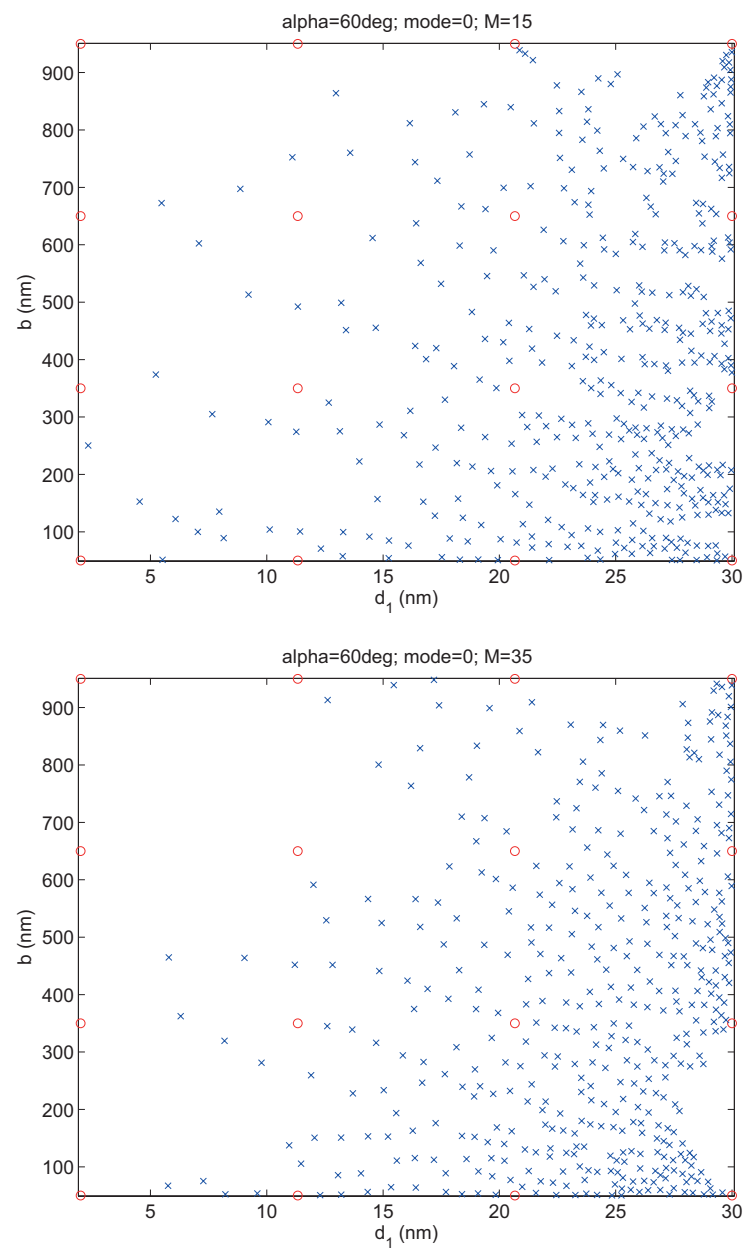

Fig. 4 Mapping for $b=330 \mathrm{~nm}, d_{1}=22 \mathrm{~nm}, \alpha=60$, for a different number of Fourier coefficients.

In contrast to a three-region model detailed in Subsection 3.1, we have four regions where two of them are of finite thickness in between semi-infinite regions of air (1) and silicon (4). Using the fact that there is no periodicity in the $\mathrm{z}$ direction, we get matrix $\mathrm{W}$ in the following form:

$$
\mathbf{W}=\mathbf{B}^{(12)} \mathbf{U}^{(2)}\left(\bar{d}_{2}\right) \mathbf{B}^{(23)} \mathbf{U}^{(3)}\left(\bar{d}_{3}\right) \mathbf{B}^{(34)} .
$$

Here we can see, that of the two finite thickness layers, the first (2) requires the permittivity tensor (basically a Heaviside $\Pi$ function) to be expanded into a Fourier series - this can easily be done analytically. The second (3) layer is homogeneous, and in the case of a scalar permittivity, also isotropic. In this case no Fourier expansion has to be done, or, equivalently, we calculate the Fourier series of a constant function $\vec{\varepsilon}_{3}(\mathbf{r})=\varepsilon_{3}$.

A standard null-zone ellipsometer measures two angles, $\Psi$ and $\Delta$. They can be connected to our model as follows:

$$
\rho=\frac{r_{p p}}{r_{s s}}=\tan (\Psi) \exp (\jmath \Delta),
$$

where $r_{s s}$ is the amplitude reflection coefficient connecting the incident $T E$ and reflected $T E$ waves, and $r_{p p}$ is amplitude reflection coefficient connecting the incident $T M$ and reflected TM waves [22].
We can find $\rho$ for each Fourier-mode of the reflected light:

$$
\rho_{i}=\frac{r_{p p, i}}{r_{s s, i}}
$$

Where $i=-M, \ldots, M$ is the inspected Fourier-mode and $M$ is the order of the Fourier expansion.

\section{Numerical examples}

To illustrate the results of the theory outlined herein, a couple of numerical studies are presented in this section.

First, the convergence of the simulation with respect to the degree of the Fourier expansion is qualitatively studied in Fig. 4. We can see that the number of Fourier coefficients considered does not significantly alter the distribution of values in the input space $\left(d_{1}, b\right)$; the simulator converges with increasing $M$. Further calculations are performed by setting order as $M=25$.

Second, the sample pattern of some optimal databases $\mathbb{D}$ are presented for different configurations in Fig. 5. Since the databases are equidistant in the output space, the larger the density of points in a region of the input space, the larger the precision of the inversion. This can be understood the following way: if we consider two neighboring points barely distinguishable during a measurement in the output space, then the same can 

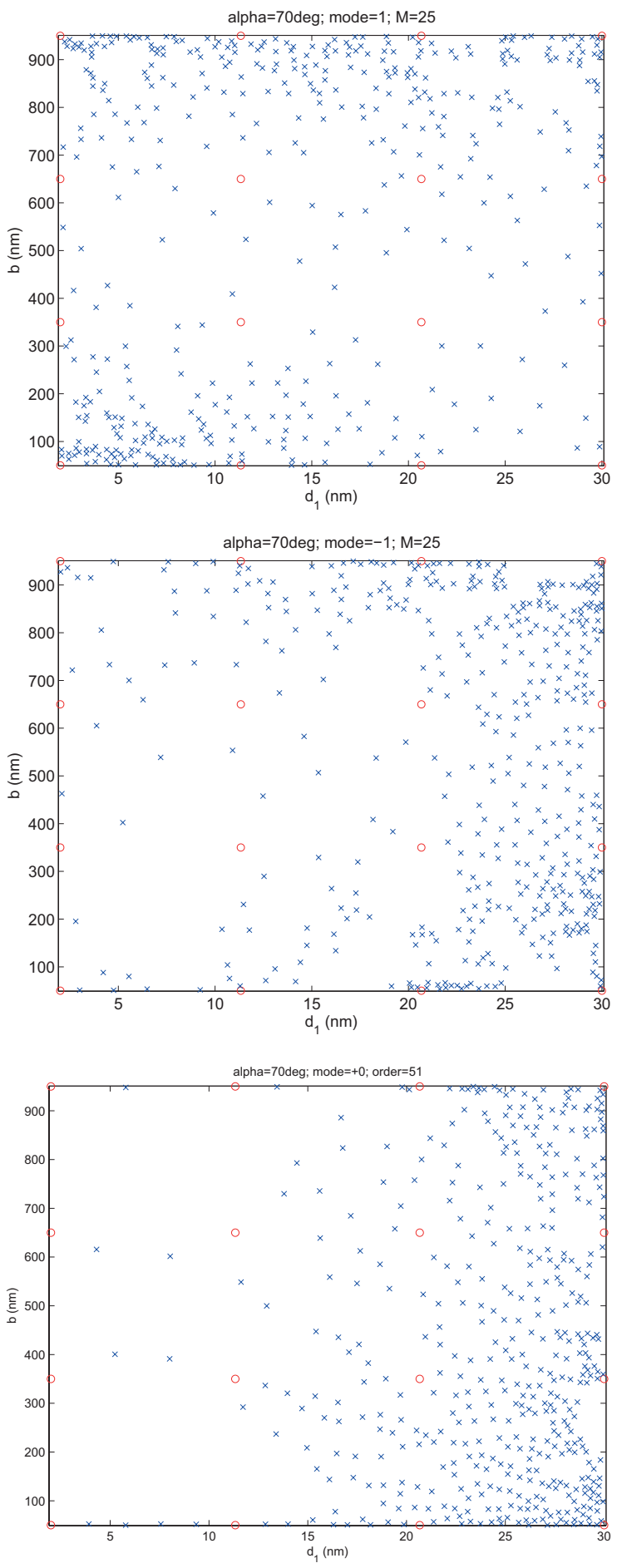
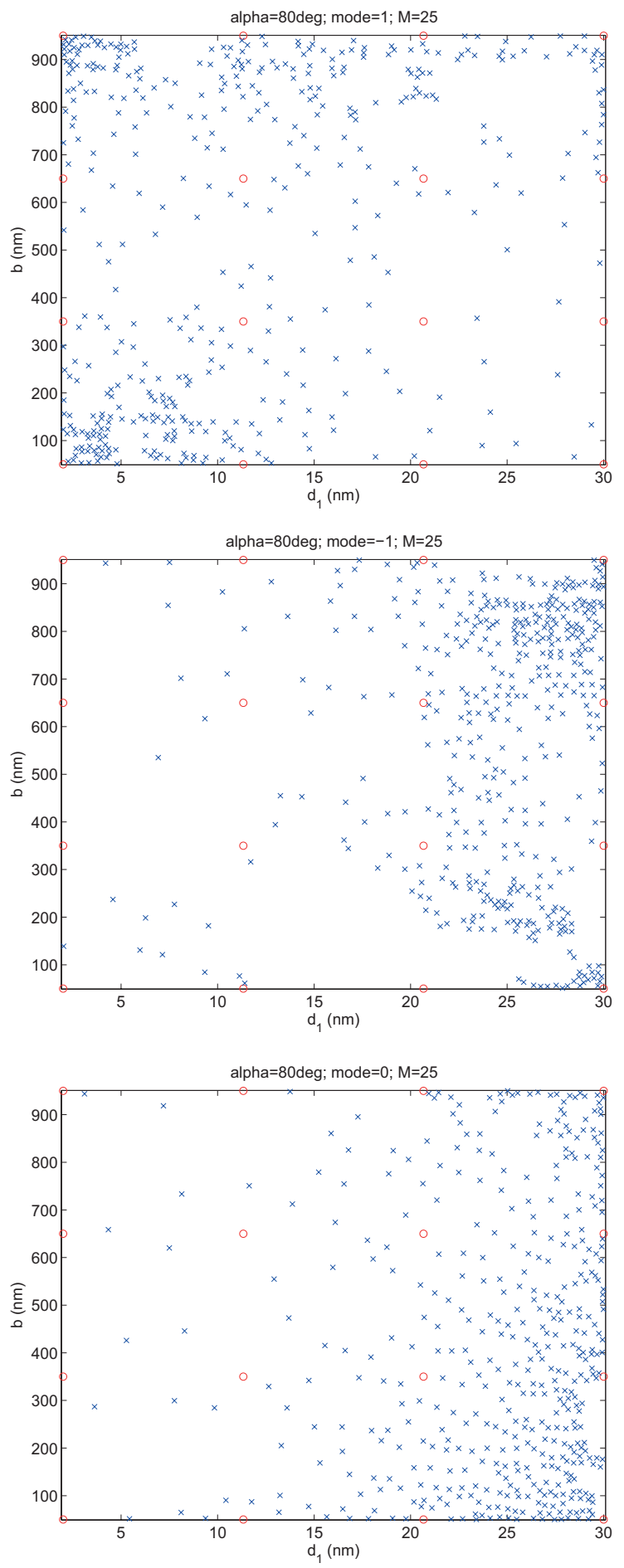

Fig. 5 Distribution of states for the first, zeroth and minus first diffraction orders, at $\alpha=70^{\circ}$, and $\alpha=80^{\circ}$, degrees of incidence.

be said after inversion about the corresponding points in the input space. For a region $\mathbf{R}_{1}$ with a small density of states, two neighbors are far apart, and the expected accuracy of the solution to the inverse problem is smaller. On the other hand, in a region $\mathbf{R}_{2}$ with a larger density of states, the distance between neighbors in the input space is smaller, so we can solve the inverse problem with a larger accuracy. It is said that the problem is well conditioned in $\mathbf{R}_{2}$ and ill conditioned in $\mathbf{R}_{1}$.

One could try to explain the zeroth order distributions as follows. Let us consider a grating with $d_{1}=0$. Since the periodicity of such a grating can be considered infinite, only the zeroth diffraction order can be observed. Let us assume that $d_{2}$ is sufficiently large so that all incident light is absorbed before reaching the boundary of the silicon and permalloy regions. In this case, a small change in $d_{2}$ will not result in any change of the reflected light. On the contrary, if $d_{2}$ is sufficiently small, even a small change in it will have an effect on the reflected light due to secondary reflection from the permalloy-silicon boundary (see Fig. 6).

In our case, where $d_{1} \neq 0$, there are obviously other effects at play, but the general character of the distribution is not 
inconsistent with the explanation presented above - where thickness of the surface layer increases, the density of states in the zeroth diffraction order decreases.

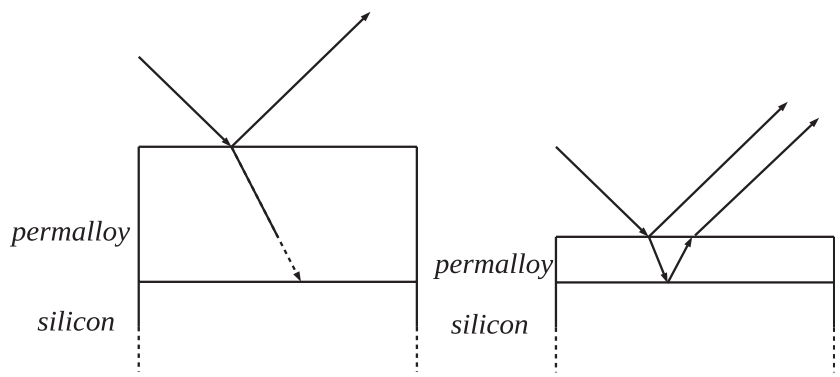

Fig. 6 Explaining the zeroth order distributions.

Thirdly, some quantitative analysis is presented in Table 1. The true (to be found) input parameters are chosen such that they represent locations in the input space with high and low sample density, i.e., well-conditioned and ill-conditioned regions in the sense of the inverse problem, while the first one is chosen to model the geometry described in [18]. In this comparison, the simplex method is used to solve the optimization problem (1). The noise function has a parameter of 2.0 degrees, the fit was performed for 20 virtual experiments in all cases.
Points $(5 \mathrm{~nm}, 200 \mathrm{~nm})$ and $(27 \mathrm{~nm}, 250 \mathrm{~nm})$ for $\alpha=70^{\circ}$ demonstrate this quite well. While the first diffraction order is better conditioned for the former and a worse for the latter, in the case of the minus first order the opposite is true. A similar comparison can be seen in the case of zeroth order $(26 \mathrm{~nm}, 100 \mathrm{~nm})$ and $(5 \mathrm{~nm}, 200 \mathrm{~nm})$ at 60 degrees of incidence. Fits were performed for the dimensions of the grating studied in $[14,18]$ as well.

Finally, some results of inversion by means of nearest neighbor interpolation are shown in Fig. 7. Herein a fine grid defines a database and repeated simulations are done, each using noisecorrupted data of a given probability distribution. Let us note that the same noise level causes significantly different uncertainty of the solution for different gratings. This is consistent with the conclusion drawn from the sample density of optimal databases.

\section{Conclusion}

In this work, it was proven that the method used in EddyCurrent nondestructive Testing can also successfully characterize the inverse problem in diffraction gratings' critical dimension measurement, thus give an insight to the precision of the measurements depending on the grating parameters' location in the input field of grating dimensions. This is numerically demonstrated in Table 1 and graphically in Fig. 7.

Table 1 Fits performed for the virtual experiments.

\begin{tabular}{|c|c|c|c|}
\hline$b=330 \mathrm{~nm}, d_{1}=22 \mathrm{~nm}, \alpha=60^{\circ}$ & 0th o.o.d & -1st o.o.d. & 1st o.o.d. \\
\hline$b[\mathrm{~nm}]$ & 337.93 & 330.15 & 329.35 \\
\hline$\delta_{b}[\mathrm{~nm}]$ & 16.19 & 0.92 & 1.93 \\
\hline$d_{1}[\mathrm{~nm}]$ & 22.13 & 22.01 & 21.94 \\
\hline$\delta_{d_{1}}[\mathrm{~nm}]$ & 0.27 & 0.07 & 0.18 \\
\hline$\alpha=60^{\circ}$, 0th o.o.d. & \multicolumn{2}{|c|}{$b=100 \mathrm{~nm}, d_{1}=26 \mathrm{~nm}$} & $b=250 \mathrm{~nm}, d_{1}=5 \mathrm{~nm}$ \\
\hline$b[\mathrm{~nm}]$ & \multicolumn{2}{|c|}{103.91} & 229.15 \\
\hline$\delta_{b}[\mathrm{~nm}]$ & \multicolumn{2}{|c|}{6.52} & 53.83 \\
\hline$d_{1}[\mathrm{~nm}]$ & \multicolumn{2}{|c|}{26.05} & 5.43 \\
\hline$\delta_{d_{1}}[\mathrm{~nm}]$ & \multicolumn{2}{|c|}{0.14} & 0.75 \\
\hline$\alpha=70^{\circ}, 1$ th o.o.d. & \multicolumn{2}{|c|}{$b=200 \mathrm{~nm}, d_{1}=5 \mathrm{~nm}$} & $b=250 \mathrm{~nm}, d_{1}=27 \mathrm{~nm}$ \\
\hline$b[\mathrm{~nm}]$ & \multicolumn{2}{|c|}{199.77} & 250.16 \\
\hline$\delta_{b}[\mathrm{~nm}]$ & \multicolumn{2}{|c|}{0.51} & 3.30 \\
\hline$d_{1}[\mathrm{~nm}]$ & \multicolumn{2}{|c|}{4.99} & 27.01 \\
\hline$\delta_{d_{1}}[\mathrm{~nm}]$ & \multicolumn{2}{|c|}{0.04} & 0.15 \\
\hline$\alpha=70^{\circ},-1$ st o.o.d. & \multicolumn{2}{|c|}{$b=200 \mathrm{~nm}, d_{1}=5 \mathrm{~nm}$} & $b=250 \mathrm{~nm}, d_{1}=27 \mathrm{~nm}$ \\
\hline$b[\mathrm{~nm}]$ & \multicolumn{2}{|c|}{199.91} & 250.26 \\
\hline$\delta_{b}[\mathrm{~nm}]$ & \multicolumn{2}{|c|}{6.42} & 0.52 \\
\hline$d_{1}[\mathrm{~nm}]$ & \multicolumn{2}{|c|}{5.11} & 26.99 \\
\hline$\delta_{d_{1}}[\mathrm{~nm}]$ & \multicolumn{2}{|c|}{0.39} & 0.03 \\
\hline$b=330 \mathrm{~nm}, d_{1}=22 \mathrm{~nm}, 0$ th o.o.d. & \multicolumn{2}{|c|}{$\alpha=70^{\circ}$} & $\alpha=80^{\circ}$ \\
\hline$b[\mathrm{~nm}]$ & \multicolumn{2}{|c|}{330.70} & 328.98 \\
\hline$\delta_{b}[\mathrm{~nm}]$ & \multicolumn{2}{|c|}{7.53} & 6.34 \\
\hline$d_{1}[\mathrm{~nm}]$ & \multicolumn{2}{|c|}{22.06} & 22.03 \\
\hline$\delta_{d_{1}}[\mathrm{~nm}]$ & \multicolumn{2}{|c|}{0.18} & 0.17 \\
\hline
\end{tabular}


alpha $=80 \mathrm{deg} ;$ mode $=-1 ; M=25 ; \sigma_{n}=0.001$

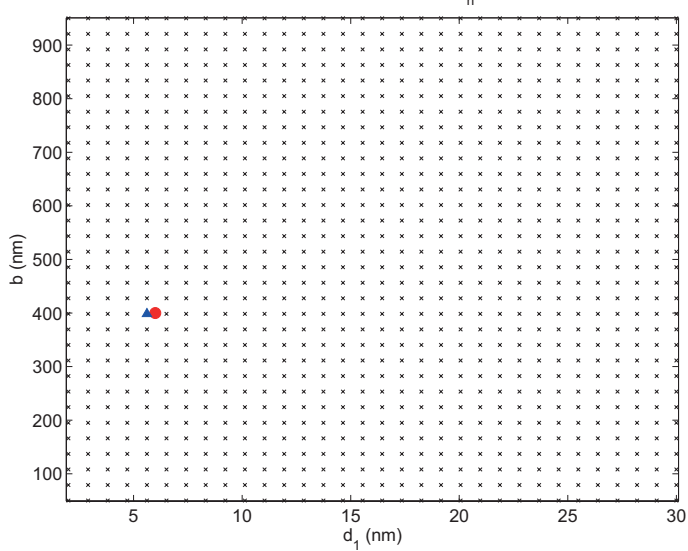

alpha $=80$ deg; mode $=-1 ; M=25 ; \sigma_{n}=0.01$

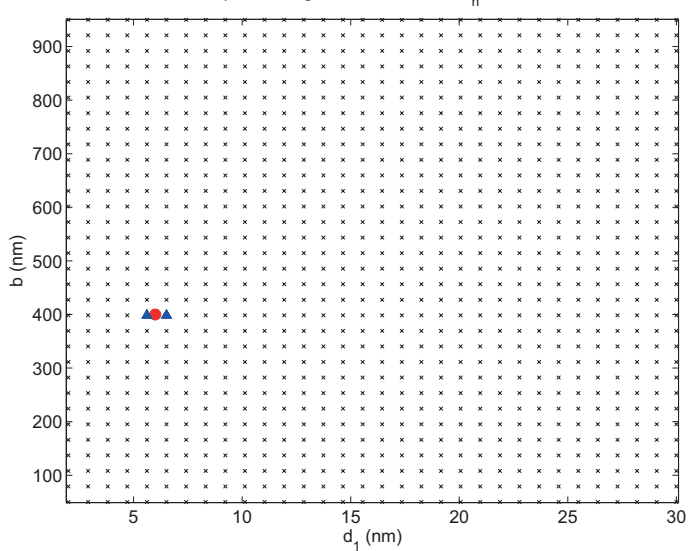

alpha=80deg; mode $=-1 ; M=25 ; \sigma_{n}=0.1$

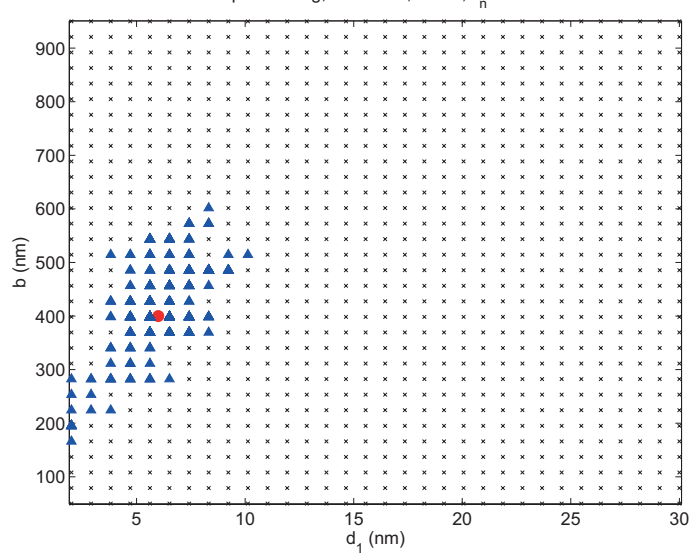

alpha=80deg; mode $=-1 ; \mathrm{M}=25 ; \sigma_{\mathrm{n}}=0.5$

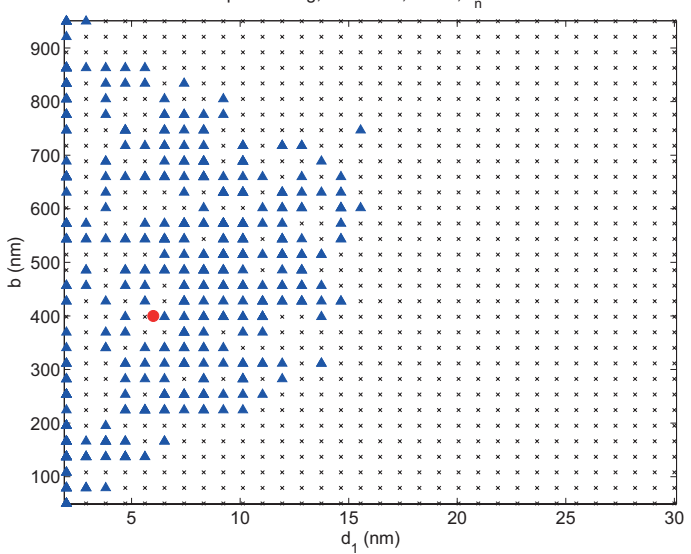

alpha $=80 \mathrm{deg} ;$ mode $=-1 ; M=25 ; \sigma_{n}=0.001$

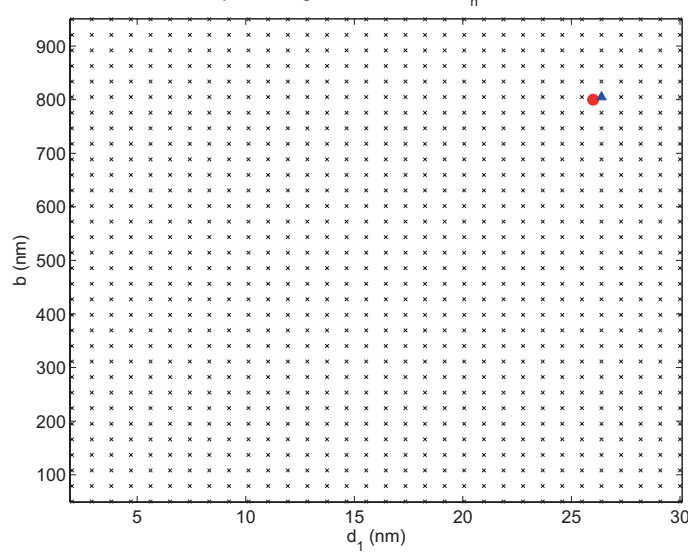

alpha=80deg; mode $=-1 ; M=25 ; \sigma_{n}=0.01$

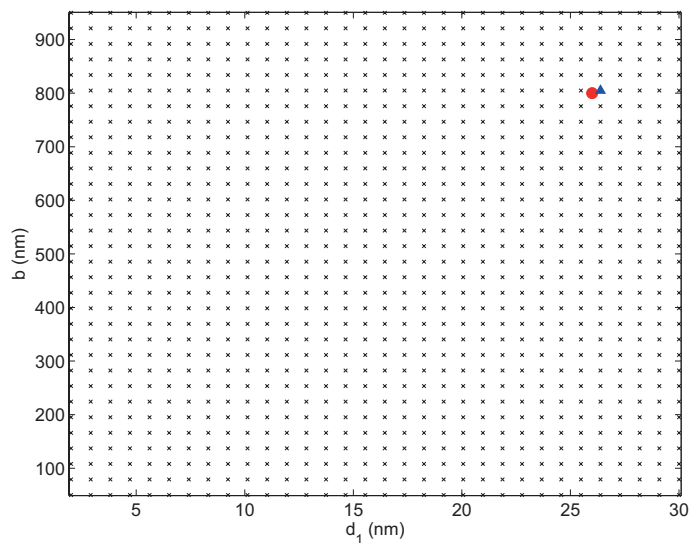

alpha $=80 \mathrm{deg} ;$ mode $=-1 ; \mathrm{M}=25 ; \sigma_{\mathrm{n}}=0.1$

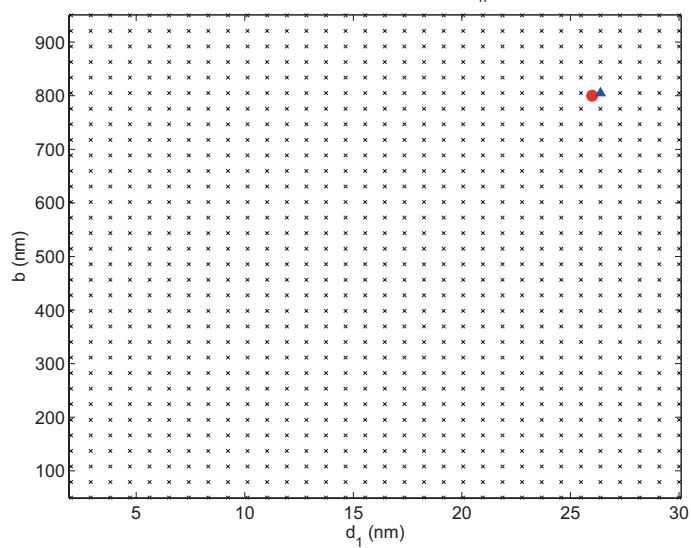

alpha=80deg; mode $=-1 ; M=25 ; \sigma_{n}=0.5$

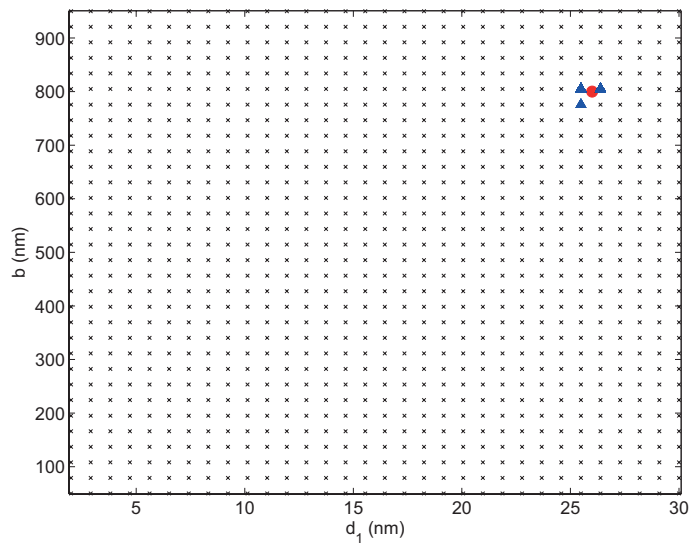

Fig. 7 Results of Nearest Neighbor interpolation on a regular grid at different levels of measuerement noise ( $\sigma_{n}^{14}:$ standard deviation of Gaussian white noise). -: true parameter, $\boldsymbol{\Delta}$ : reconstructed parameter using the noise-corrupted data (test of 500 runs). 
It can also be seen that fits in the minus first and first diffraction orders tend to have a smaller deviation value than the zeroth order. To what extent this is true, and to precisely describe this phenomenon, more information is needed about the measurement's details; this may be an interesting topic for further research. A change in the angle of incidence did not cause qualitative differences in the distribution of states, although there are certain cases where the choice of angle might be significant [24].

With further work, it seems possible that a method could be devised to choose an appropriate measurement setting (diffraction order, angle of incidence), based on the material parameters and the approximate location of the grating's critical dimensions in the input field.

\section{References}

[1] Goodno, G. D., Dadusc, G., Miller, R. J. D. "Ultrafast heterodyne-detected transient-grating spectroscopy using diffractive optics." Journal of the Optical Society of America B. 15(6), pp. 1791-1794. 1998.

DOI: $10.1364 /$ josab.15.001791

[2] Kong, S. H., Wijngaards, D. D. L., Wolffenbuttel, R. F. "Infrared microspectrometer based on a diffraction grating." Sensors and Actuators A: Physical.92(1-3), pp. 88-95. 2001. DOI: 10.1016/s0924-4247(01)00544-1

[3] Mansuripur, M. "Classical optics and its applications." Cambridge University Press, 2002.

[4] Palmer, C. A., Loewen, E. G. "Diffraction grating handbook." Newport Corporation Springfield, Ohio, USA, 2005.

[5] Harvey, K. C., Myatt, C. J. "External-cavity diode laser using a grazingincidence diffraction grating." Optics Letters. 16(12), pp. 910-912. 1991. DOI: $10.1364 /$ ol.16.000910

[6] Chen, J., Bos, P. J., Vithana, H., Johnson, D. L. "An electro-optically controlled liquid crystal diffraction grating." Applied Physics Letters. 67(18), pp. 2588-2590. 1995. DOI: 10.1063/1.115140

[7] Antos, R., Pistora, J., Mistrik, J., Yamaguchi, T., Yamaguchi, S., Horie, M., Visnovsky, S., Otani, Y. "Convergence properties of critical dimension measurements by spectroscopic ellipsometry on gratings made of various materials." Applied Physics Letters. 100(5), 054906. 2006. DOI: $10.1063 / 1.2337256$

[8] Antos, R., Mistrik, J., Yamaguchi, T., Visnovsky, S., Demokritov, S. O., Hillebrands, B. "Evaluation of the quality of permalloy gratings by diffracted magneto-optical spectroscopy." Optics Express. 13(12), pp. 4651-4656. 2005. DOI: 10.1364/opex.13.004651

[9] Antos, R., Mistrik, J., Yamaguchi, T., Visnovsky, S., Demokritov, S. O., Hillebrands, B. "Evidence of native oxides on the capping and substrate of permalloy gratings by magneto-optical spectroscopy in the zerothand first-diffraction orders." Applied Physics Letters. 86(23), 231101. 2005. DOI: $10.1063 / 1.1944904$

[10] Antos, R. "Diffraction on laterally structured anisotropic periodic systems." 2006. [Online]. Available from: https://is.cuni.cz/webapps/zzp/ download/140027455

[11] Varga, G. "Determination of debye-waller factors from elastic diffraction peaks of thermal energy atomic scattering from solid surfaces." Vacuum. 50(3-4), pp. 339-342. 1998. DOI: 10.1016/s0042-207x(98)00064-5

[12] Moharam, M. G., Gaylord, T. K. "Coupled-wave analysis of reflection gratings." Applied Optics. 20(2), pp. 240-244. 1981. DOI: $10.1364 /$ ao. 20.000240

[13] Veis, M., Antos, R., Visnovsky, S., Kulkarni, P., Venkataramani, N., Prasad, S., Mistrik, J., Krishnan, R. "Complete permittivity tensor in sputtered CuFe204 thin films at photon energies between 2 and $5 \mathrm{eV}$." Materials. 6(9), pp. 4096-4108. 2013. DOI: 10.3390/ma6094096
[14] Veis, M., Antos, R. "Advances in optical and magnetooptical scatterometry of periodically ordered nanostructured arrays." Journal of Nanomaterials. 2013, 2013. DOI: 10.1155/2013/621531

[15] Blitz, J. "Electrical and Magnetic Methods of Non-destructive Testing." Springer Netherlands, 1997. DOI: 10.1007/978-94-011-5818-3

[16] Bilicz, S., Lambert, M., Gyimóthy, Sz. "Kriging-based generation of optimal databases as forward and inverse surrogate models." Inverse Problems. 26(7), 074012. 2010. DOI: 10.1088/0266-5611/26/7/074012

[17] Bilicz, S., Lambert, M., Gyimothy, S., Pavo, J. "Solution of inverse problems in nondestructive testing by a kriging-based surrogate model." IEEE Transactions on Magnetics. 48(2), 495-498. 2012.

DOI: $10.1109 /$ tmag.2011.2172196

[18] Marak, K. "Optics of periodic nanostructures." 2013. [Online]. Available from: https://is.cuni.cz/webapps/zzp/detail/128070?lang=en

[19] Jellison, G. E. "Data analysis for spectroscopic ellipsometry." Thin Solid Films. 234(1-2), pp. 416-422. 1993. DOI: 10.1016/0040-6090(93)90298-4

[20] mathworks. fmincon. 2016. [Online]. Available from: http://www.mathworks.com/help/optim/ug/fmincon.html

[21] Loewen, E. G., Popov, E. "Diffraction gratings and applications." CRC Press, 1997.

[22] Rokushima, K., Antos, R., Mistrík, J., Visnovský, S., Yamaguchi, T. "Optics of anisotropic nanostructures." Czechoslovak Journal of Physics. 56(7), pp. 665-764. 2006. DOI: 10.1007/s10582-006-0128-0

[23] Visnovsky, S. "Optics in magnetic multilayers and nanostructures." CRC Press, 2006.

[24] Antos, R., Mistrik, J., Yamaguchi, T., Veis, M., Liskova, E., Visnovsky, S., Pistora, J., Hillebrands, B., Demokritov, S. O., Kimura, T., Otani, Y. "Magneto-optical spectroscopic scatterometry for analyzing patterned magnetic nanostructures." Journal of the Magnetics Society of Japan. 30(6-2), pp. 630-636. 2006. DOI: 10.3379/jmsjmag.30.630

[25] Antos, R. "Fourier factorization with complex polarization bases in modeling optics of discontinuous bi-periodic structures." Optics Express. 17(9), pp. 7269-7274. 2009. DOI: 10.1364/oe.17.007269

[26] Antos, R., Veis, M. "Fourier factorization with complex polarization bases in the plane-wave expansion method applied to two-dimensional photonic crystals." Optics Express. 18(26), pp. 27511-27524. 2010. DOI: 10.1364/oe.18.027511

[27] Azzam, R. M. A., Bashara, N. M., Ballard, S. S. "Ellipsometry and polarized light." Physics Today. 31(11), 72, 1978. DOI: 10.1063/1.2994821

[28] Granet, G., Guizal, B. "Efficient implementation of the coupled-wave method for metallic lamellar gratings in TM polarization." Journal of the Optical Society of America A." 13(5), pp. 1019-1023. 1996. DOI: 10.1364 josaa.13.001019

[29] Hecht, E., Zajac, A. "Optics." 2002.

[30] Kneubühl, F. "Diffraction grating spectroscopy." Applied Optics. 8(3), pp. 505-519. 1969. DOI: 10.1364/ao.8.000505

[31] Lalanne, P. "Improved formulation of the coupled-wave method for two-dimensional gratings." Journal of the Optical Society of America A. 14(7), pp. 1592-1598. 1997. DOI: 10.1364/josaa.14.001592

[32] Lalanne, P., Morris, G. M. "Highly improved convergence of the coupledwave method for TM polarization." Journal of the Optical Society of America A. 13(4), pp. 779-784. 1996. DOI: 10.1364/josaa.13.000779

[33] Moharam, M. G., Gaylord, T. K. "Rigorous coupled-wave analysis of planar-grating diffraction." Journal of the Optical Society of America. 71(7), pp. 811-818. 1981. DOI: 10.1364/josa.71.000811

[34] Schnopper, H. W., Van Speybroeck, L. P., Delvaille, J. P., Epstein, A., Källne, E., Bachrach, R. Z., Dijkstra, J., Lantward, L. "Diffraction grating transmission efficiencies for XUV and soft x rays." Applied Optics. 16(4), pp. 1088-1091. 1977. DOI: 10.1364/ao.16.001088 